\title{
Cirurgia de varizes: história e evolução
}

\author{
Varicose vein surgery: history and evolution
}

\author{
Charles Angotti Furtado de M edeiros*
}

\begin{abstract}
Resumo
Como a safenectomia é uma cirurgia bem estabelecida, a utilização de um método pouco invasivo para obter os mesmos efeitosindica que os ben efícios esperados a curto e médio prazo devem prevalecer sobre os riscos previstos. Entretanto, em se tratando de uma doença muito prevalente na população mundial, há grande preocupação por parte dos órgãos de saúde governamentais e das sociedades científicas correlacionadas sobre esse assunto, de tal forma que os argumentos sobre novas modalidades de tratamento devem estar bem fundamentados, inclusive para aceitação pelo público em geral.

Com o intuito de se avaliar efetivamente novos dispositivos, devem ser conduzidos estudos em pacientes e com grupos controles que sejam bem delineados. É preciso submeter qualquer projeto de pesquisa sobre 0 assunto à apreciação do comitê de ética na instituição em que o trabalho será conduzido para aprovação prévia.
\end{abstract}

Palavras-chave: Insuficiência venosa, tratamento, fotocoagulação a laser.

\section{H istória da cirurgia de varizes}

Desde a Antigüidade, são encontrados relatos a respeito de varizes dos membros inferiores (M M II), conformedocumentado no papiro deEbers, do reinado deAmenophisl (1550 a.C.). N a G réciaAntiga, réplicas de M M II com varizes eram oferecidas aos deuses nos templos com o intuito de se conseguir alívio dos sintomas $^{1}$. T ambém há muito se escreve sobre o tratamento cirúrgico de varizes. Existem relatos de que $\mathrm{H}$ ipócrates

\footnotetext{
* Mestre, Especialista em Angiorradiologia e Cirurgia Endovascular, SBACV. Membro, Centro de Referência em Cirurgia Endovascular, Universidade Estadual de Campinas (UNICAMP), Campinas, SP. Professor, Disciplina de Cirurgia Vascular Periférica, Faculdade de Ciências Medicas, UNICAMP, Campinas, SP.

Artigo submetido em 24.08.06, aceito em 21.11.06.
}

J Vasc Bras 2006;5(4):295-302.

Copyright $\odot 2006$ by Sociedade Brasileira de Angiologia e de Cirurgia Vascular.

\begin{abstract}
Since great saphenous vein stripping is a well established surgery, the use of a minimally invasive method to obtain the same effects indicates that short and medium-term benefits should prevail over expected risks. However, because it is a very common disease in the world population, there is great concern about this subject by government health institutions and correlated scientific societies. Therefore, the arguments about new treatment modalities must be well based, including to gain acceptance by the general public.

To effectively evaluate new devices, there must be well designed clinical studies with groups and controls. All projects about this matter must be submitted to the appreciation of an ethics committee at the institution where the research will be carried out for previous approval.
\end{abstract}

Keywords: Venous insufficiency, treatment, laser photocoagulation.

(460-377 a.C.) cauterizavavarizes com ferro em brasa². Ele foi o primeiro a notar a associação entre as veias varicosaseasúlceras na perna. $\mathrm{H}$ ipócrates aindacitaem suas obras, várias vezes, as doenças venosas dos M M II.

D urante a Era Romana ${ }^{3}$, Aurelius Cornelius C elsius (53 a.C.-7 d.C.) descreveu com detal hes a realização de uma exérese de varizes. Ele fazia incisões escalonadas, cauterizava a veiaeretiravaa quantidadedevasos que era possível, de forma não muito diferente das incisões escal onadas nas cirurgias venosas hoje praticadas. Aurelius Cornelius C elsus também aconselhou o uso de bandagens paraúlceras na perna. Pouco mais de 100 anos depois, Cláudio $\mathrm{G}$ aleno (130-200 d.C.) extirpava as veias dilatadas com um gancho entre duas ligaduras e aplicava vinho às feridas. A ele é atribuída a invenção da ligadura cirúrgica, sem a qual a cirurgianão teria se desenvolvido. 
A Escola de Alexandria foi o maior centro de estudos médicos no mundo antigo. Fundada por Alexandre, o Grande, atraiu muitos pesquisadores e estudiosos, entre eles médicos de todo 0 mundo. $N$ esse centro deexcelência, jásefalava em ligadurasvasculares, e o precursor na ligadura das veias varicosas como tratamento foi o médico bizantino Aetius de Amida (502-575) nas margens do Tigret.

No século 7, Paulus Aegineta (607-690 d.C.), que também foi aluno em Alexandria, descreveu o primeiro relato sobre a ligadura da veia safena interna (VSI) na coxa. Ele comprimia a veia acima e abaixo e, quando a veia estava visível, marcava-a com uma tinta especial. Então, praticava a excisão da veia marcada, ligando o coto proximal e o distal ${ }^{3}$. Interessante lembrar que essa técnica semelhante foi empregada vários séculos após pelo célebre cirurgião francês Ambroise Paré (15101590).

Ao fim do Império Romano do 0 cidente, seguiu-se o radicalismo religioso que desencadeou os 1.000 anos de trevas da I dade M édia. A medicina que havia sido iniciada na M esopotâmia e na civilização egípcia e depois desenvolvida na Era C lássica grecoromana estava agora estagnada. Baseado na teoria humoral de Galeno, ainda se acreditava que os males estagnados eram expelidos através das úlceras e, por isso, estas não deveriam ser curadas sob risco de causar sérias doenças.

Sendo assim, pouco progresso ocorreu até a época do Renascimento. $\mathrm{N}$ o começo dessa era, que mudou de forma tão importante as artes, a medicina ainda não conhecia a circulação sangüínea, e persistiam as idéias antigas. I sso durou atéa descoberta das válvulas venosas e o seu papel na circulação, por W illiam H arvey, que se tornaram públicas em 1628. Somente nos estudos de Richard W iseman, em 1676, ficou demonstrado que a incompetência valvular resultava da dilatação de uma veia. Assim, apesar dos conhecimentos desdeostempos de $\mathrm{H}$ ipócrates, ele foi o primeiro a considerar que a úlcera era o resultado direto de um defeito circulatório, cunhando o termo úlcera varicosa ${ }^{4}$.

N o final do século 18 e início do 19, os cientistas, influenciados pelos princípios de N ewton (1687), passaram a considerar como fator importante a atuação da gravidade no retorno venoso, constituindo assim uma nova linha de pensamento - a teoria mecânica. $N$ essa época, Petit e, depois, $\mathrm{H}$ unter indicavam o repouso no leito em posição horizontal como conduta inicial. Bünger, em 1823, praticou a primeira operação de enxerto de pele bem-sucedida no ser humano, com a intenção de cicatrizar uma ferida 5 .

Finalmente, em 1868, as investigações científicas de John Gay apontaram que poderiam existir outras condições mais severas afetando também o sistema venoso profundo (SVP), quando descreveu as alterações da síndrome pós-trombótica ea anatomia das veias perfurantes no tornozelo. Eleentão introduziu o termo úlcera venosa.

Com a introdução das técnicas de assepsia e a descoberta da anestesia no final do século 19, o tratamento cirúrgico também evoluiu6,7. Em 1885, M adelung descreveu a retirada completa da VSI combinada com a ligadura de veias tributárias. W illiam M oore, em 1896, realizou, na Austrália, a ligadura da crossa da VSI sob anestesia local ${ }^{3}$. A grande mudança viria com a descrição por M ayo (1904) de um instrumento metálico em forma de anel para extração extraluminal da $\mathrm{VSI}^{8}$. Keller (1905), no ano seguinte, utilizou um dispositivo intraluminal, constituído deestiletedemetal torcido que invertia a veia à medida que a tracionava ${ }^{9}$, sendo queBabcock (1907) desenvolveu o fleboextrator, que consistia de uma vareta metálica com extremidade em forma de oliva, protótipo dos materiais usados atualmente $\mathrm{e}^{10}$.

0 próximo avanço apareceu com a apresentação de H omans (1917) sobre a fisiopatologia da trombose venosa profunda. Seus estudos demonstraram que a recanalização com destruição das válvulas era muito comum e estava intimamente relacionada com 0 aparecimento das úlceras nos membros afetados.

No entanto, em meados de 1920, a injeção de substâncias esclerosantes nas veias varicosas tornou-se muito popular, e a cirurgia de varizes só foi revitalizada com a operação de Linton (1938) sobre a ligadura das veias perfurantes insuficientes ${ }^{11}$.

Com o tempo, firmou-se o conceito da necessidade de interrupção dos pontos de refluxo venoso com a fleboextração das veias safenas interna e externa, a ligadura das veias perfurantes insuficientes e a ressecção escalonada das tributárias varicosas, conhecida como cirurgia radical de varizes.

O utra importante contribuição foi a publicação por M uller, já em 1966, da técnica conhecida como miniflebectomia, que consiste na retirada de tributárias varicosas com instrumentos delicados, semeIhantes às agulhas de crochê, diminuindo assim o trauma cirúrgico e proporcionando um excelente resultado estético ${ }^{12,13}$. 
0 tratamento cirúrgico das al terações do SVP também começou a ser divulgado em meados de 1940, com 0 aparecimento de várias técnicas para abordagem da insuficiência venosa crônica (IVC). I nicialmente eram realizadas ligaduras das veias do SVP (veia femoral e, posteriormente, veia poplítea). Sabe-se que hoje tais métodos foram abandonados porque, além de serem ineficientes, podem piorar ainda mais o quadro de hipertensão venosa. Porém, em 1958, Palma \& Esperon obtiveram sucesso com o enxerto fêmoro-femoral cruzado para tratar uma veia ilíaca ocluída e, no ano de 1968, Kistner realizou a primeira reconstrução valvular, dando início a uma nova era na abordagem de um antigo problema14,15.

Apesar de terem surgido na mesma época que as técnicasparaligadura das veias do SV $P$, osprocedimentos que visavam tratar as veias perfurantes insuficientes na perna começaram a ganhar espaço somente 1 década depois. A operação consistia basicamente na ligadura subaponeurótica de várias veias perfurantes através de incisão longitudinal na perna ${ }^{16}$, tentando-se evitar a área tecidual comprometida pelas alterações tróficas existentes, sendo as mais conhecidas a incisão medial (Linton) já citada, a incisão medial distal (Cockett) ea incisão posterior (Felder). A ligaduraendoscópicasubfascial das veias perfurantes foi descrita por dois autores, H auer \& Sattler, na Alemanha em 1985 e, depois, adaptada ao aparato habitual da videocirurgia ${ }^{17}$. Com a vantagem de ser uma técnica menos invasiva que as anteriores, não prescinde de equipamentos especiais e de pessoal devidamente treinado.

\section{A física do laser}

Apesar de os cientistas e filósofos debaterem 0 conceito da luz desde cerca de 4.000 anos a.C., as primeirasteoriassignificativasnão foram desenvolvidas atéa metade final do século 17. A teoria corpuscular foi originalmente proposta por I saac N ewton, que dizia que as leis da mecânica aplicam-se a quase todas as características daluz. Q uasenamesmaépoca, C hristian $\mathrm{H}$ uygens formulou a teoria da onda de luz. Ele afirmava, acertadamente, quea luz deveria atravessar um meio denso de forma mais lenta do que no ar, contrariando asidéias deN ewton. $D$ evido ao pensamento dominante na comunidade científica daquela época, o conceito corpuscular permaneceu a teoria dominante ${ }^{18}$.

Q uase 100 anos depois, os experimentos de Foucault $\&$ M ichelson concluíram que a velocidade da luz em um meio mais denso era real mentemaislenta do que ao atravessar 0 ar. Como conseqüência, a concepção de $\mathrm{N}$ ewton sobre 0 assunto caiu no esquecimento, e a teoria da onda deluz passou a ser aceita. Foi somenteem 1887 que $\mathrm{H}$ eirich $\mathrm{H}$ ertz demonstrou todas as características observacionais do feixe de luz como uma forma de energia eletromagnética, mas ainda existiam contradições nessa nova teoria. As respostas só surgiriam no início do século seguinte, com a teoria quântica da estrutura atômica e o conceito da emissão da luz.

Em seus estudos, $\mathrm{N}$ iels Bohr visualizou a estrutura atômica de forma similar à estrutura do universo e postulou queoselétrons de cada átomo existiam apenas em órbitas específicas ao redor do núcleo central. M ax Planck e Albert Einstein propuseram que a emissão de radiação ocorre quando um elétron salta de uma órbita de maior energia para outra de menor energia. Este foi o primeiro passo em direção à concepção do laser. Experimentosem 1921 por H . A. Compton isolaram as colisões entre esses elétrons. D escobriu-se que eles se comportavam como matéria e obedeciam às leis clássicas de conservação de energia e momento. A teoria da luz havia voltado ao conceito anterior da teoria corpuscular proposta por N ewton em 1667.

A essa altura, já estava bem estabelecido que a luz certamente se comportava como um fenômeno de onda, e os cientistas foram colocados na berlinda mais umavez. 0 mistério danatureza dupla daluz (partículaonda) ainda não está completamente resolvido. Apesar da contradição aparente, esses dois conceitos têm uso prático na descrição do comportamento do feixe deluz: enquanto a teoria quântica (partícula) é utilizada para descrever os fundamentos dos processos de emissão e absorção que ocorre nos átomos e moléculas, a teoria eletromagnética (onda) é mais apropriada na discussão da propagação da radiação entre a fonte e o receptor.

Assim, a história do laser ${ }^{19}$ começou mesmo pela formulação desua teoria, em 1917, por Albert Einstein, que definiu o conceito de "emissão estimulada de radiação". Após a Segunda Guerra M undial, com o avanço da el etrônica, foi desenvolvido o M ASER, processo de amplificação de microondas por emissão de radiação estimulada.

0 primeiro laser - palavra formada pelas iniciais de light amplification by stimulated emission of radiation foi apresentado em 1960 por Theodore $\mathrm{H}$ arold M aiman, na Califórnia. Ele utilizava um pequeno cilindro de cristal de rubi sintético que, depois de excitado por feixes de luz curtos e intensos, emitia pulsos com 
comprimento de onda de $690 \mathrm{~nm}$, um raio luminoso próximo à faixa do vermel ho no espectro visível. 0 laser de rubi desenvolvido apresentava propriedades desconhecidas até então.

O laser possui a particularidade de ser um feixe de luz coerente, isto é, monocromático e unidirecional. Significa quetoda a energia emitida por elecaminha em sentido paralelo e sem dispersão da luz, podendo ser concentrada em um único ponto. Contudo, diferentes tipos de laser têm diferentes comprimentos de onda e, sendo assim, cada um del es possui aplicações especiais.

A maioria dos aparel hos delaser écomposta por um material ativo (sólido ou gasoso) acoplado a um espel ho refletor em cada extremidade. U tilizando o fornecimento de energia extrínseca (descarga elétrica), os átomos presentes no material ativo mudam de órbita em um processo conhecido como excitação. Passado certo tempo, osátomos excitados voltam à sua órbita habitual, situada em um nível mais baixo, liberando um quantum de energia - o fóton. Este, por sua vez, chocasecom outro átomo excitado, produzindo um efeito em cascata. U m grande número de fótons carregados é emitido e se refletem para trás e para frente pelos espelhosatésejuntarem eserem expelidosatravés deum pequeno orifício em uma das extremidades desta cavidade ressonante. D esta forma, é produzida uma luz muito intensa de bilhões de $\mathrm{W}$ atts $/ \mathrm{cm}^{2}$.

Já em 1961, apareciam os primeiros estudos da aplicação do laser em biologia. D aí em diante, viria a se desenvolver o seu uso em microfotometria, microscopia eletrônica, citologia, genética, fotobiologia e outras áreas. 0 primeiro simpósio sobre a recente criação foi em 1964, em Boston. D esde então, apareceram várias experiências com o uso do laser em medicina. Logo surgiu a idéia de utilização dessa tecnologia especificamenteem cirurgia. A nova perspectivaestava baseadana realização de procedimentos menos traumáticos, com pouca ou nenhuma tração tecidual. Entretanto, os primeiros aparelhos decepcionaram quanto à sua aplicação, apesar do furor na época do seu invento, e o progresso só apareceu no início da década de 1970 com a construção do laser cirúrgico a $\mathrm{CO}_{2}$ por Polanyi ${ }^{19}$.

0 laser gera um feixe de luz com intensidade enorme, que é altamente colimado e, como resultado, representa um ponto focal bem preciso. A precisão também é uma das necessidades das cirurgias mais delicadas. Adicionalmente, uma das dificuldades principais em cirurgia éa hemostasia; então, se o laser pode prevenir ou minimizar o sangramento, ele deverá ser de grande valia. Assim, têm-se duas propriedades chaves que sugerem que o laser pode servir como valoroso instrumento cirúrgico: a precisão e a diminuição da perda sangüínea.

\section{Cirurgia minimamente invasiva}

As tentativas de obliteração minimamente invasiva da VSI iniciaram com a invenção da seringa no século 19. D esde então, as técnicas que utilizam líquido esclerosante para o tratamento da insuficiência venosa vieram evoluindo. Porém, somente em 19440 rbach descreveu o método que combina ar à escleroterapia.

No início, a escleroterapia foi extensamente usada como um substituto para a cirurgia, mas depois de um estudo prospectivo, randomizado por $\mathrm{H}$ obbs, na década de 1970, o interesse diminuiu. E assim, durante a última metade do século 20, a escleroterapia como tratamento principal das veias varicosas veio e foi embora. Porém, logo antes do início do século 21, um novo método desenvolvido por T essari renovou 0 assunto, dando força às recentes publicações de $C$ abrera a respeito da injeção de polidocanol na forma de microespuma guiada pelo ultra-som (US), conhecida como ecoesclerose ${ }^{20-22}$.

A possibilidade de destruição da parede vascular através da coagulação foi descrita ${ }^{23}$ na década de 1950. Em uma outra linha de pesquisa, originada na França, surgiu a idéia de aplicar a criocirurgia no tratamento das varizes dos M M II ${ }^{24}$. A partir daí, obteve-se um aumento abundante nas observações clínicas e experimentais sobre 0 efeito das baixas temperaturas no sistema vascular25-29.

$M$ ais recentemente, deu-se início à aplicação da cauterização endoluminal das veias safenas com a radiofreqüência (RF), que começou a ser publicada $\mathrm{a}^{30-32}$ a partir de1999. N osúltimosanos, uma nova técnica usando energia a laser mostrou resultados promissores no tratamento de varizes dos M M II. A fotocoagulação pelo laser endovenoso foi descrita 33,34 no mesmo ano que a RF. Alguns dos dispositivos disponíveis no mercado, inclusive, já tiveram sua aprovação homologada pelo FD A (Food and Drug Administration) nos EUA para esse fim.

\section{Conceitos atuais}

0 padrão-ouro para o tratamento cirúrgico de varizes devido à insuficiência da junção safeno-femoral 
(JSF) associada ao refluxo na VSI éa ligadura da crossa com fleboextração 35-38.

Como a safenectomia é uma cirurgia bem estabelecida, a utilização de um método pouco invasivo para obter os mesmos efeitos indica que os benefícios esperados a curto e médio prazo devem prevalecer sobre os riscos previstos. $\mathrm{No}$ entanto, em se tratando de uma doença muito prevalente na população mundial, há grande preocupação por parte dos órgãos de saúde governamentais e das sociedades científicas correlacionadas sobre esse assunto ${ }^{39}$. Portanto, os argumentos sobre novas modalidades de tratamento devem estar bem fundamentados, inclusive para aceitação pelo público em geral, visto o fácil acesso aos meios de comunicação em massa presente nestes dias.

As cirurgias pouco invasivas não dispensam uma boa avaliação pré-operatória e o preparo adequado do paciente a ser operado ${ }^{40}$. N o que se refere ao uso de novas tecnologias, o médico tem a obrigação de explicar os detalhes do procedimento, bem como sua indicação, vantagens e as possíveis complicações inerentes ao método. M esmo assim, os pacientes deveriam ser tratados somente após assinarem o consentimento pós-informação.

Além da anamnese e do exame físico, os exames complementares são de importância fundamental para avaliação do sistema venoso dos membros inferiores $^{41}$. A ausculta com aparelho de Doppler de onda contínua é o primeiro e mais simples exame a ser realizado. Porém, o mapeamento duplex - ou duplex scan, como é conhecido - é hoje o exame complementar deescolha no diagnóstico etratamento da insuficiência venosa dos M M II 42-48.

Após a observação de que a pressão medida na veia safena (pressão venosa ambulatorial) diminuía durante 0 exercício, esta passou a ser usada como parâmetro na avaliação de pacientes com IVC 49 . Porém, pelo seu caráter invasivo e pouco prático, tornou-se difícil fazer repetidas avaliações com esse sistema para estudar os pacientes submetidos a cirurgia de varizes. Ao assumir queo volume desanguenas veias éresponsável pela pressão venosa, pode-se dizer que a pressão venosa é determinada pelo volume venoso. D esta forma, a pletismografia ganhou espaço na avaliação e no seguimento desses pacientes por ser uma técnica mais simples e que mede as variações de volume ocorridas nas extremidades em resposta à mudança de postura e ao exercício muscular ${ }^{50-54}$.
O uso da pletismografia a ar (PG A) tornou-se cada vez mais popular em razão da disposição de aparelhos comerciais no mercado. N o entanto, al gumas críticas devem ser levadas em consideração. Q uando o teste é repetido em um mesmo paciente, pode haver variações nos valores obtidos, o que indica que este é um exame improvável para detectar pequenas mudanças nos parâmetros analisados ${ }^{55}$. T ambém éfato quealgunspacientes não colaboram ou não conseguem completar os exercícios adequadamente. A pesar de não invalidarem o exame, tais aspectos devem ser levados em consideração em qualquer estudo no qual a PG A éutilizada como teste.

A flebografiafoi, durantemuitosanos, considerada padrão no estudo das doenças venosas ${ }^{43}$. D evido ao desconforto provocado pelo exameassociado aos riscos de um método invasivo e ao grande avanço na propedêutica não-invasiva, ela passou a ser pouco utilizada. Atualmenteestá reservada ao estudo deanomalias venosas, fístula arteriovenosa, diagnóstico diferencial entre varizes primárias e secundárias e no planejamento préoperatório das reconstruções venosas.

Esses conhecimentos são essenciais para o cirurgião vascular e permitem que cada paciente receba uma terapêutica individualizada. Sendo assim, os objetivos do tratamento cirúrgico da insuficiência venosa primária seriam: a) Primo non nocere; b) retirada das varizes; c) melhora funcional; d) satisfação estética; e) evitar as recidivas; f) preservar as veias safenas quando possível.

C om a descrição, em 1949, da utilização da VSI como enxerto arterial ${ }^{56}$, a sua extração, na cirurgia de varizes, passou a ser contestada por muitos cirurgiões ${ }^{57-60}$. Entretanto, quando há indícios de comprometimento mais grave das veias safenas, dispõe-se hoje de várias opções para a remoção destas da circulação venosa (T abela 1).

U m dos axiomas básicos no estudo de todas as possibilidades de aplicação do laser é: "se você não precisadele, não use". I sso também seaplicaao emprego de outras tecnologias recentes em medicina, nas quaisa primeira consideração é determinar se há necessidade definitiva para a suautilização. A simples respostaéque, apesar de as novas técnicas cirúrgicas, incluindo o laser endovenoso, ainda não terem atingido o estágio da perfeição, el astêm encontrado numerosas aplicaçõesno campo da cirurgia vascular e se mostram como uma grande promessa para o futuro ${ }^{61-63}$. Portanto, o cirur- 
Tabela 1 - T écnicas disponíveis atual mente para o tratamento das veias safenas

\begin{tabular}{ll}
\hline Preservação da veia safena & D esconexão das tributárias varicosas apenas \\
\hline Fleboextração convencional & Parcial \\
& T otal \\
Crossectomia & Ligadura da VSI na JSF sem fleboextração \\
Valvuloplastia da crossa & Plicatura da válvula da JSF \\
Escleroterapia guiada pelo US & Punção cutânea \\
& Injeção por cateter \\
Energia eletromagnética & Laser endovenoso \\
& Radiofreqüência bipolar \\
\hline
\end{tabular}

JSF = junção safeno-femoral; US = ultra-som; VSI = veia safena interna.

gião, que está sempre procurando mel horar suatécnica, agora dispõe dessas novas tecnologias. A questão é: elas têm algum valor?

A preocupação a ser considerada é o instrumental sofisticado e caro exigido por muitos desses procedimentos. Atualmente, esse fator limita em muito o uso em larga escala de novas tecnologias no Brasil. 0 assunto exerce um enorme fascínio, inerente às novas tendências que aparecem na mídia. Rodeado de uma publicidade excessiva, os perigos da exploração indiscriminada são óbvios.

Entretanto, se há a possibilidade para o uso dessas novas modalidades de tratamento, a próxima consideração a ser feita é determinar se são seguras. Contudo, nem sempre é possível prever o que acontecerá aos humanos quando se utilizam sistemas de pesquisa que testam animais como modelo. T ambém são necessários programas desegurança previamenteplanejados para as investigações clínicas.

Ainda com relação aos aspectos éticos, não se pode esquecer que qualquer trabalho científico a ser desenvolvido sobre este assunto deve seguir as normas estabelecidas pela Resolução 196/96 do Conselho N acional de Saúde e as diretrizes recomendadas pela C omissão $\mathrm{N}$ acional de Ética em Pesquisa.

\section{C onclusão}

Com intuito de se avaliar efetivamente novos dispositivos, devem ser conduzidos estudos em pacientes e com grupos controles que sejam bem delineados. É preciso submeter o projeto de pesquisa à apreciação do comitê de ética na instituição em que o trabalho será conduzido para aprovação prévia.

\section{R eferências}

1. Bettmann OL. A pictorial history of medicine. Springfield: Charles C. Thomas; 1979. p. 14-31.

2. H ipocrates. The genuine works of $\mathrm{H}$ ippocrates. Baltimore: W illiams \& Wilkins; 1946. p. 325-36.

3. Rose SS. Historical development of varicose vein surgery. In: Bergan JJ, Goldman MP, editors. Varicose veins and telangiectasias. Saint Louis: Q uality M edical Publishing; 1993. p. 123-47.

4. Anning ST . The historical aspects. In: D odd H, C ockett FB. The pathology and surgery of the veins of the lower limb. 2nd ed. Edinburgh: Churchill Livingstone; 1976. p. 3-17.

5. Spina V. Cirurgia plástica. In: RaiaAA, ZerbineEJZ, editors. Clínica cirúrgica Alípio Corrêa N etto. São Paulo: Sarvier; 1994.

6. Edwards EA. The treatment of varicose veins. Surg G ynecol O bst. 1934;59:916-28.

7. Summers J. E. H ighlightsin thetreatment of varicoseveinsand ulcers. Am J Surg. 1953;86:443-63.

8. Mayo CH. The surgical treatment of varicose veins. St Paul M ed J. 1904;6:695.

9. KellerW L. A new method of extirpating theinternal saphenous and similar veins in varicose conditions: a preliminary report. NY M ed J 1905;82:385-6.

10. Babcock WW . A new operation for theextirpation of varicose veins of the leg. NY M ed J. 1907;86:153-6.

11. Linton RR. Thecommunicating veins of the lower leg and the operative technique for their ligation. Ann Surg. 1938;107: 582-93. 
12. M uller R. Traitment des varices par le phlébectomie ambulatoire. Phlebologie. 1966;19:277-9.

13. Kafejian 0 , 0 liveira $G A C$, T akayanagi $T$. I novações técnicas na cirurgia de varizes visando a resultados estéticos. R ev Ass M ed Bras. 1976;22:296-7.

14. Kistner RL. Surgical repair of the incompetent femoral vein valve. Straub Clin Proc. 1968;34:41-3.

15. Eklof BG, Kistner RL, M asuda EM . V enous bypass and valve reconstruction: long-term efficacy. V asc M ed. 1998;3:157-64.

16. Robison JG, Elliott BM , Kaplan AJ. Limitations of subfascial ligation for refractory chronic venous stasis ulceration. Ann V asc Surg. 1992;6:9-14.

17. $H$ auer $G$, Bergan $J$ J, W erner $A, M$ itterhusen $M, N$ asralla $F$. $D$ evelopment of endoscopic dissection of perforating veins and fasciotomy for treatment of chronic venous insufficiency. Ann Vasc Surg. 1999;13:357-64.

18. Goldman L, Rockwell JR. The history and physics of laser emission. In: Goldman L, Rockwell JR. Lasers in medicine. N ew York: G ordon \& Breach Science; 1991. p. 7-40.

19. Ferrari BT . Raio laser: uma técnica que conquista a medicina. Rev Bras Clin Ter. 1986;15:188-91.

20. Cabrera J, Redodo P, Becerra A, et al. Ultrasound-guided injection of polidocanol microfoam in the management of venous leg ulcers. Arch D ermatol. 2004;140:667-73.

21. Coleridge Smith P. Saphenous ablation: sclerosant or sclerofoam? Semin Vasc Surg. 2005;18:19-24.

22. Bergan J,PascarellaL, M ekenasL. V enousdisorders: treatment with sclerosant foam. J Cardiovasc Surg (Torino). 2006;47: 9-18.

23. Sawyer PN, Pate JW . Bioelectric phenomena as an etiologic factor in intravascular thrombosis. Am J Physiol. 1953;175: 103-7.

24. M illeret $R$, Le Pivert $P$. C ryosclerosis of the saphenous veins in varicose reflux in the obese and elderly. Phlebologie. 1981;34:601-5.

25. Le Pivert P. Controlled cryosurgery of varices of the lower extremities. A new therapeutic approach. Apropos of 350 cases. Phlebologie. 1987;40:123-48.

26. Cheatle TR, Kayombo B, Perrin M. Cryostripping the long and short saphenous veins. Br J Surg. 1993;80:1283.

27. Garde C. Cryosurgery of varicose veins. J Dermatol Surg O ncol. 1994;20:56-8.

28. Etienne G, Constantin JM, H evia M. Cryo-stripping: an advance in the treatment of varicose veins. 3811 operated limbs. Presse M ed. 1995;24:1017-20.

29. Constatin J M , Etienne G, H evia M. T echnique and results of cryo-stripping in the treatment of varicose veins of the lower limbs. Ann Chir. 1997;51:745-8.

30. W eissRA, Goldman M P. C ontrolled RF-mediated endovenous shrinkage and occlusion. In: G oldman M P, W eissRA, Bergan JJ. V aricoseveins\& telangiectasias: diagnosisand management. 2nd ed. Q uality M edical Publishing; 1999. p. 217-24.

31. Chandler JG, Pichot O, SessaC, Schuller-Petrovic S, Kabnick LS, Bergan JJ. T reatment of primary venous insufficiency by endovenous saphenous vein obliteration. V asc Endovascular Surg. 2000;34:201-14.

32. Goldman M P. Closure of the greater saphenous vein with endoluminal radiofrequency thermal heating of the vein wall in combination with ambulatory phlebectomy: preliminary 6-month follow-up. Dermatol Surg. 2000;26:452-6.
33. Boné $C$. T ratamiento endoluminal de las varices con láser de diodo. Estudio preliminar. Rev Patol Vasc 1999;5:35-46.

34. N avarro L, M in RJ, BonéC. Endovenouslaser: anew minimally invasi ve method of treatment for varicose veins - preliminary observations using an $810 \mathrm{~nm}$ diode laser. Dermatol Surg. 2001;27:117-22.

35. ProebstleTM, Lehr H A, K argl A, et al. Endovenoustreatment of the greater saphenous vein with a 940-nm diode laser: thrombotic occlusion after endoluminal thermal damage by laser-generated steam bubbles. J V asc Surg. 2002;35:729-36.

36. Proebstle TM, Sandhofer M , Kargl A, et al. Thermal damage of the inner vein wall during endovenous laser treatment: key role of energy absorption by intravascular blood. Dermatol Surg. 2002;28:596-600.

37. Sybrandy JE, Wittens $\mathrm{CH}$. I nitial experiences in endovenous treatment of saphenous vein reflux. J V asc Surg. 2002;36: 1207-12.

38. Perkowski P, Ravi R, Gowda RC, et al. Endovenous laser ablation of the saphenous vein for treatment of venous insufficiency and varicose veins: early results from a large single-center experience. J Endovasc Ther. 2004;11:132-8.

39. M affei $F H A$. Insuficiência venosa crônica: diagnóstico e tratamento clínico. In: M affei FH A, Lastória S, Yoshida W B, editores. D oenças vasculares periféricas. 2. ed. Rio de Janeiro: M edsi; 1995. p. 1003-1011.

40. G aspar RJ, M edeiros CAF. T ratamento combinado dacirurgia de varizes com a escleroterapia detelangiectasias dos membros inferiores no mesmo ato. J Vasc Bras. 2006;5:53-7.

41. Luccas GC, M edeiros CAF. Úlcera varicosa - tratamento cirúrgico. In: M erlo I, Parente JBH, Komlos PP, editores. $V$ arizes e telangiectasias: diagnóstico e tratamento. Rio de Janeiro: Revinter; 2006. p. 325-34.

42. C astro eSilva M, C abral ALS, BarrosJ r. N , C astro AA, Santos $M E R C$. D iagnóstico etratamento da doença venosa crônica. J V asc Bras. 2005;4:S185-94.

43. N eglén $P$, Raju S. A comparison between descending phlebography and duplex Doppler investigation in the evaluation of reflux in chronic venousinsufficiency: achallenge to phlebography as the "gold standard". J Vasc Surg. 1992;16:687-93.

44. Luccas GC, N agase $Y, M$ enezes $F H$, et al. Cirurgia de varizes dos membros inferiores: avaliação pré-operatória do sistema venoso com mapeamento duplex. Cir V asc Angiol. 1996;12: 15-20.

45. N eglén P, Egger JF 3rd, O livier J, Raju S. H emodynamic and clinical impact of ultrasound-derived venousrefluxparameters. J V asc Surg. 2004;40:303-10.

46. Thomaz JB, Thomaz YCM . Cirurgia da safena parva. Rev Angiol Cir Vasc 2004;3:111-20.

47. O liveira A, Vidal EA, França GJ, Toregiani J, Timi JRR, M oreiraRCR. Estudo das variaçõesanatômicas daterminação da veia safena parva pelo eco-D oppler colorido. J V asc Bras. 2004;3:223-30.

48. França GJ, Timi JRR, Vidal EA, Oliveira A, Secchi $F$, M iyamotto M. O eco-Doppler colorido na avaliação das varizes recidivadas. J V asc Bras. 2005;4:161-6.

49. França $L H G, T$ avares $V$. Insuficiência venosa crônica. U ma atualização. J Vasc Bras. 2003;2:318-28. 
50. K atzM L, C omerotaA , Kerr R. Air plethysmograph (APG ${ }^{T M}$ ): a new technique to evaluate patients with chronic venous insufficiency. J Vasc T ech. 1991;15:23-7.

51. W elkieJF, Kerr RP, Katz M L, ComerotaAJ . C an noninvasive venous volume determinations accurately predict ambulatory venous pressure? J Vasc Tech. 1991;15:186-90.

52. EvangelistaSSM. Pletismografiano estudo das doenças venosas. In: M affei FH A, Lastória S, Y oshida W B, Rollo H A, editores. D oenças vasculares periféricas. Rio de Janeiro: M edsi; 2002. p. 479-92.

53. Evangelista SSM . Pletismografia a ar e laboratório vascular. In: M erlo I, Parente JBH, Komlos PP, editores. Varizes e telangiectasias: diagnóstico e tratamento. Rio de Janeiro: Revinter; 2006. p. 137-47.

54. Engelhorn CA, B effa CV, Bochi G, Pullig RC, Cassou M F, Cunha SS. A pletismografia a ar avalia a gravidade da insuficiência venosa crônica?J V asc Bras. 2004;3:311-6.

55. Yang D, Vandogen YK, Stacey M C. V ariability and reliability of air plethysmographic measurements for the evaluation of chronic venous disease. J V asc Surg. 1997;26:638-42.

56. Kunlin J. Le traitement de l'arterite obliterante par la greffe veineuse. Arch M al Coeur. 1949;42:371-2.

57. Luccas GC, Parente JBF, N agase $Y$, Lane JC. Preservação da veia safena magna em cirurgia de varizes: resultados tardios. Cir V asc Angiol. 1995;11:15-8.

58. Luccas G C, N agase Y, SilveiraSAF, Potério Filho J. A valiação quantitativa do refluxo na veia safena interna com duplex: aplicação no planejamento cirúrgico das varizes primárias dos membros inferiores. Cir V asc Angiol. 1999;15(Supl):27S.
59. Luccas $G C, N$ agase $Y$, Silveira SAF, M enezes FH . M edida do diâmetro e refluxo da veia safena interna no pré e pósoperatório dacirurgia devarizes. Cir Vasc Angiol. 2001;17:24.

60. Engelhorn CA, Engelhorn AL, Cassou M F, Zanoni CC, Gosalan CJ, Ribas E. Classificação anatomofuncional da insuficiênciadasveiassafenasbaseadano eco-D oppler colorido, dirigida para o planejamento da cirurgia de varizes. J V asc Bras. 2004;3:13-9.

61. M edeirosCAF. Estudo comparativo entre o laser endovenoso e a fleboextração convencional da veia safena interna em pacientes com varizes primárias [dissertação]. Campinas: U niversidade Estadual de Campinas; 2005.

62. de M edeiros CA, Luccas GC. Comparison of endovenous treatment of an $810 \mathrm{~nm}$ laser versus conventional stripping of the great saphenous vein in patients with primary varicose veins. D ermatol Surg. 2005;31:1685-94.

63. M edeirosCAF. T ratamento cirúrgico endovascular com laser. In: M erlo I, Parente JBH, Komlos PP, editores. Varizes e telangiectasias: diagnóstico e tratamento. Rio de Janeiro: Revinter; 2006. p. 288-95.

\section{Correspondência:}

Charles Angotti Furtado de $M$ edeiros

Rual zabel N egrão Bertotti, 101/52 - Bairro M . Sto. Antônio CEP 13087-508 - Campinas, SP

Tel.: (19) 3256.9771

E-mail: drcharlesangotti@hotmail.com 